\title{
Lunar near surface plasma environment from Chandrayaan-2 Lander platform: RAMBHA-LP payload
}

\author{
G. Manju*, Tarun K. Pant, P. Sreelatha, Santhosh J. Nalluveettil, \\ P. Pradeep Kumar, Nirbhay Kumar Upadhyay, Md. Mosarraf Hossain, \\ Neha Naik, Vipin Kumar Yadav, Rosmy John, R. Sajeev, Jothi Ramalingam, \\ Philip George, Amarnath Nandi, N. Mridula, Aswathy R. P. Janmejay Jaiswal \\ Rana, Snehil Srivastava and Satheesh Thampi
}

Vikram Sarabhai Space Centre, ISRO, Thiruvananthapuram 695 022, India

\begin{abstract}
The near surface lunar plasma environment is modulated by important components like the photoelectron sheath, solar wind, lunar surface potential, etc. In situ measurements of lunar near surface plasma are not available as of now. Previous lunar missions which explored the near surface environment have arrived at estimates of lunar photo electron densities mainly from lunar sample returns. The Chandrayaan-2 lunar mission affords a unique opportunity to explore the near surface lunar plasma environment from the lunar lander platform. A Langmuir probe is developed indigenously for probing the tenuous lunar near surface plasma environment from the top deck of the lunar lander. The probe is designed to cater to a wide dynamic range of $10 /$ ce to $10,000 /$ cc. The probe behaviour is characterized in the ambient room conditions using a current source. The sensitivity of the probe to incoming ionized species is also characterized in a vacuum chamber. The Langmuir probe response is characterized such that the input current to the probe is correctly deciphered during the mission duration. The calibration of the present Langmuir probe is carried out using a standard calibrated Langmuir probe. The details of the theoretical simulations of the expected currents, the characterization and calibration activities are presented and discussed.
\end{abstract}

Keywords: Debye length, electron density, electron temperature, Langmuir probe.

Moon being the planetary body closest to Earth, has always been the object of our scientific curiosity regarding the origin of the solar system, evolution of Earth and the planetary atmospheres. Our quest regarding the existence/non-existence, origin and evolution of the planetary atmospheres, including that of Moon has been one of the major scientific endeavours of recent times. Experiments

\footnotetext{
*For correspondence. (e-mail: manju_spl@vssc.gov.in)
}

reveal that unlike Earth's dense atmosphere, Moon has a very tenuous atmosphere that is at times also termed as Moon's surface bound exosphere. It has been indicated that this tenuous lunar atmosphere also supports a tenuous plasma environment. SIDE ion detector (SIDE, Apollo 14 and 15$)^{1,2}$, the charged particle lunar environment experiment (CPLEE, Apollo 14) ${ }^{3}$, the solar wind spectrometer (SWS, Apollo 12 and 15) ${ }^{4}$ and the lunar ejecta and meteorites experiment (LEAM, Apollo 17) ${ }^{5,6}$, are some of the instruments that probed the near surface lunar environment in the 1970s. The CPLEE instrument measured a complete drop-out of electrons during a total lunar eclipse confirming that the plasma environment on the day-side lunar surface is dominated by photoelectrons ${ }^{7,8}$. Variations in the plasma environment were seen by CPLEE when the Moon was alternately in the solar wind, the terrestrial magnetotail or the terrestrial plasma sheet ${ }^{7,9}$

Plasma accumulation near the lunar surface was proposed to be caused by the possible stand-off of solar wind by the remnant magnetic field on the lunar surface ${ }^{10}$. The recent discovery of mini magnetosphere on the Moon gave a lot of credence to this proposition. The photoelectric yield measurements obtained from sample returns by the Apollo missions ${ }^{11,12}$ indicated $4.5 \mu \mathrm{A} / \mathrm{m}^{2}$ for the rate of photoelectron emission and $2 \mathrm{eV}$ for the mean kinetic energy. These values gave rise to an estimate of near surface photoelectron density of $\sim 100 / \mathrm{cc}$ and Debye length of $1 \mathrm{~m}$. The photoelectron yield measurements were combined with the high-resolution solar irradiance spectra and the photoelectron current had been estimated for three different levels of solar activity ${ }^{13}$.

The lunar near surface plasma environment is affected by the lunar surface potential which varies from 0 to $10 \mathrm{~V}$ during lunar daytime. The emission of photoelectrons from the lunar surface leaves the surface positively charged. The lunar surface potential and the electric field, $E$ (at the surface) have been estimated from different 


\section{RESEARCH ARTICLES}

plasma parameters ${ }^{13} . E^{2}$ is the proxy for dust mobilization due to electrostatic forces. This results in levitation of positively charged lunar dust, which adds a distinctly different dimension to the near surface plasma environment. The foregoing discussion shows that the near surface lunar plasma environment is replete with multiple factors which are modulated by the evolving solar conditions.

The lunar ionosphere as we know it today, is thought to be existing with electron densities of the order of $100 \mathrm{~cm}^{-3}$ below $30 \mathrm{~km}$ altitude at solar zenith angles less than $60^{\circ}$. The density reaches a peak of $\sim 400 \mathrm{~cm}^{-3}$ at around $15 \mathrm{~km}$ altitude and decreases gradually at higher altitudes and toward the surface ${ }^{14}$. The electron density enhancement appears to be confined for latitudes where solar zenith angle is below $80^{\circ}$. Despite the above scenario, several uncertainties and knowledge gaps exist. Moreover, nearly all the contemporary understanding of lunar plasma environment has come through remote sensing experiments, where it was not always easy to delineate the variability of the lunar ionosphere from the motion of the sensor. Direct measurements of the lunar plasma do not exist so far. In fact, when it comes to the measurements on lunar plasma made during different lunar missions, there is only broad agreement, leaving scope for making better and accurate measurements ${ }^{14-18}$. The radio occultation experiments have been able to give electron density measurements mostly limited to higher altitudes in the lunar ionosphere. This technique has a limitation in sampling the near surface plasma distribution. Hence other techniques have to be resorted to for sampling this region.

Although many of the Apollo missions' experiments had instruments to measure lunar plasma near the surface (below $1 \mathrm{~m}$ altitude), none of them actually made direct measurements of the electron density as discussed earlier. Conventional instruments like ionosonde are also not feasible in such tenuous environments in view of the large dimensions of antennae for the required low frequency transmission and reception. Langmuir probe is a compact instrument which has conventionally been used to measure electron density and electron temperature. The technique used herein was originally developed by Irwing Langmuir during nineteen twenties to study the plasma properties of a laboratory gas discharge ${ }^{19}$. Cylindrical Langmuir probes were used to make terrestrial ionospheric density and temperature measurements in the nineteen sixties $^{20}$. Extensive rocket-based Langmuir probe measurements of the terrestrial ionosphere have been carried out from India and abroad throughout the nineteen sixties and seventies ${ }^{21}$. A Langmuir probe can be positioned on a lunar lander to sample the near surface plasma without involving complex mechanisms.

It is in this context that the present radio anatomy of Moon bound hypersensitive atmosphere and ionospherelangmuir probe (RAMBHA-LP) payload has been proposed on board Chandrayaan-2 lander for obtaining in situ measurements of the lunar near surface plasma at an altitude of $\sim 2 \mathrm{~m}$. The primary science objectives of the RAMBHA-LP payload are: (i) to make in situ measurements of the ambient electron density/temperature near the lunar surface and (ii) to study the temporal evolution of the lunar plasma density for the first time near the surface under varying solar conditions.

\section{Langmuir probe}

This section provides the details of the instrument principle, theoretical simulations of the expected currents, the operational parameters and specifications.

\section{Instrument and principle}

Langmuir probes are valuable tools for probing plasmas in the laboratory as well as in space. An ideal plasma for investigation by the Langmuir probe is one where the electrons and ions are fully thermalized, at nearly equal temperatures, where the particle mean free path $\gg$ Debye length. A Langmuir probe consists of a conducting surface (spherical, cylindrical or planar), which is inserted into plasma and electrically biased with respect to the ground/spacecraft body to collect electron and ion currents. Using the current-voltage characteristics of the Langmuir probe, it is possible to determine the plasma density and electron temperature.

The floating potential $\left(V_{\mathrm{f}}\right)$ to a probe is the applied potential at which the ion current is balanced by the electron current resulting in zero net current collection. The space potential $\left(V_{\mathrm{s}}\right)$ or plasma potential is the potential at which the effective potential on the probe is zero. In such a case, the probe is at the potential of the plasma. The schematic of voltage-current characteristic curve for a typical Langmuir probe is given in Figure 1. The characteristic curve can be divided into three regions: (i) In the ion saturation region, for $V$ (applied potential) $<V_{\mathrm{f}}$ (the floating potential, for which the current collected is zero) the probe current is mainly a positive ion current. That is, when the probe current is at a large negative voltage, it attracts positive ions and repels electrons thereby collecting a net positive ion current. (ii) In the retarding potential region, for $V_{\mathrm{f}}$ (floating potential) $<V$ (applied potential) $<V_{\mathrm{s}}$ (the space or plasma potential), there is a predominant electron current collected in response to a retarding potential. (iii) In the electron saturation region, for $\mathrm{V}$ (applied potential) $>V_{\mathrm{s}}$ ( space potential) the positive ion current becomes negligible and current collected is a purely electron current.

The slope of the voltage versus $\log (I)$ curve in the retarding potential region is inversely related to temperature $\left(1 / T_{\mathrm{eV}}=e / k T_{\mathrm{e}}\right.$, where $T_{\mathrm{e}}$ is the electron temperature in Kelvin, $k$ the Boltzmann constant and $e$ is the electronic charge).

CURRENT SCIENCE, VOL. 118, NO. 3, 10 FEBRUARY 2020 
Thus the temperature of the electrons can be derived using this method and the thermal velocity can be estimated from the temperature using the expression ${ }^{22}$,

$$
v=\left(8 k T_{\mathrm{e}} / \pi m_{\mathrm{e}}\right)^{1 / 2},
$$

where $m_{\mathrm{e}}$ is the electronic mass.

The current $I_{0}$ due to random thermal motion of electrons is given as

$$
I_{0}=n e A v / 4 \text {, }
$$

where $n$ is the electron number density and $A$ is the probe surface area.

Equations (1) and (2) are obtained from the kinetic theory of gases.

For an electrode at a negative potential $V$ with respect to the plasma the current collected is given by ref. 22

$$
I=I_{0} \exp (e V / k T) \text {. }
$$

For a spherical electrode at a positive potential $V$ with respect to plasma, the current collected is given by ref. 22

$$
I=I_{0}(1+e V / k T) .
$$

For the ion collection mode

$$
I=I_{0}(1-e V / k T) \text {. }
$$

Expected lunar plasma environment and theoretical simulations of RAMBHA-LP characteristics for tenuous environments like lunar ambience

To design a versatile probe, theoretical simulations of LP characteristics were undertaken for a range of parameters

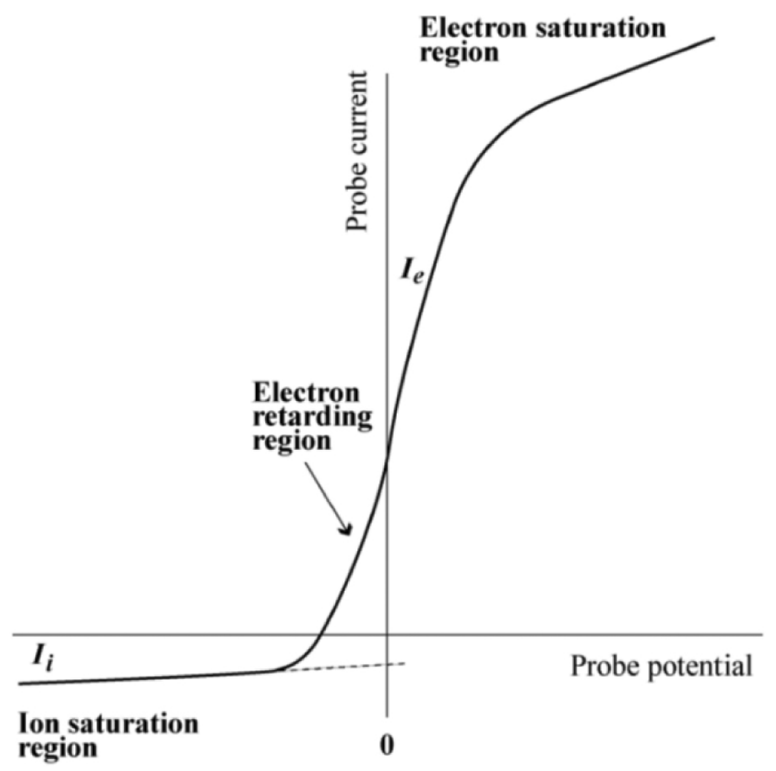

Figure 1. Ideal current voltage characteristic for Langmuir probe from introduction to space instrumentation edited by Abe and Oyama. expected in lunar ionospheric conditions. The dynamic range of the expected currents is simulated for different configurations. The RAMBHA-LP characteristics were simulated for a spherical Langmuir probe of radius, $2.5 \mathrm{~cm}$. The simulated curve for an assumed electron density of $10^{3} / \mathrm{cc}$ is shown in Figure 2. The simulations of the currents have been made for an electron density range of $10 / \mathrm{cc}$ to $10^{4} / \mathrm{cc}$. Lunar ionospheric electron density profile recently reported by Imamura et al. ${ }^{14}$ is shown in Figure 3 (dashed curve). According to them, the lowest and highest limits of the observed electron densities, at lower altitudes below $10 \mathrm{~km}$, are $10^{2} / \mathrm{cc}$ and $4 \times 10^{2} / \mathrm{cc}$ respectively considering the standard deviations as well.

In fact, the lunar plasma density is not expected to be much lower than $10^{2} / \mathrm{cc}$ as different components like photo ionization, solar wind and dust contribute to the net lunar ionization ${ }^{23}$. Nevertheless, to cater to unexpected

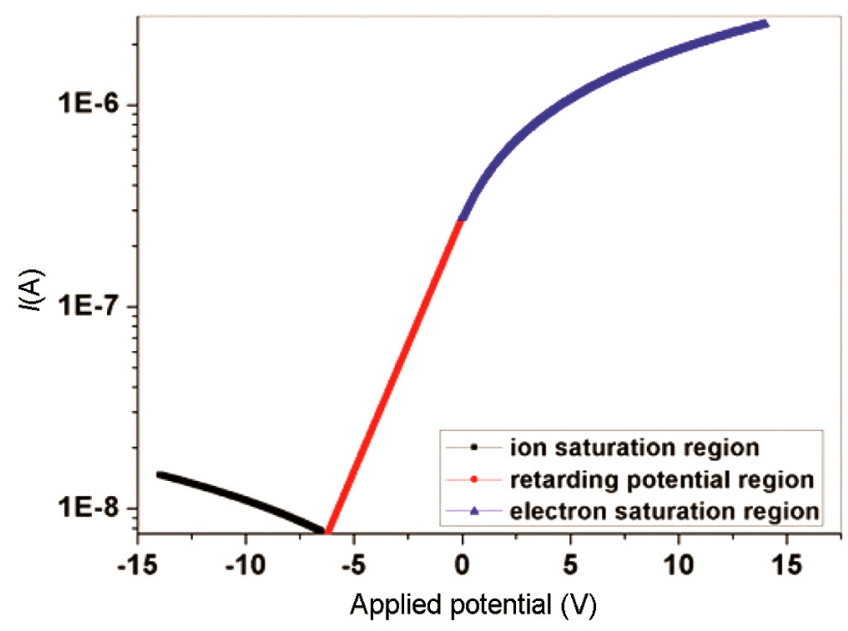

Figure 2. Simulated IV characteristic curve for lunar ionosphere.

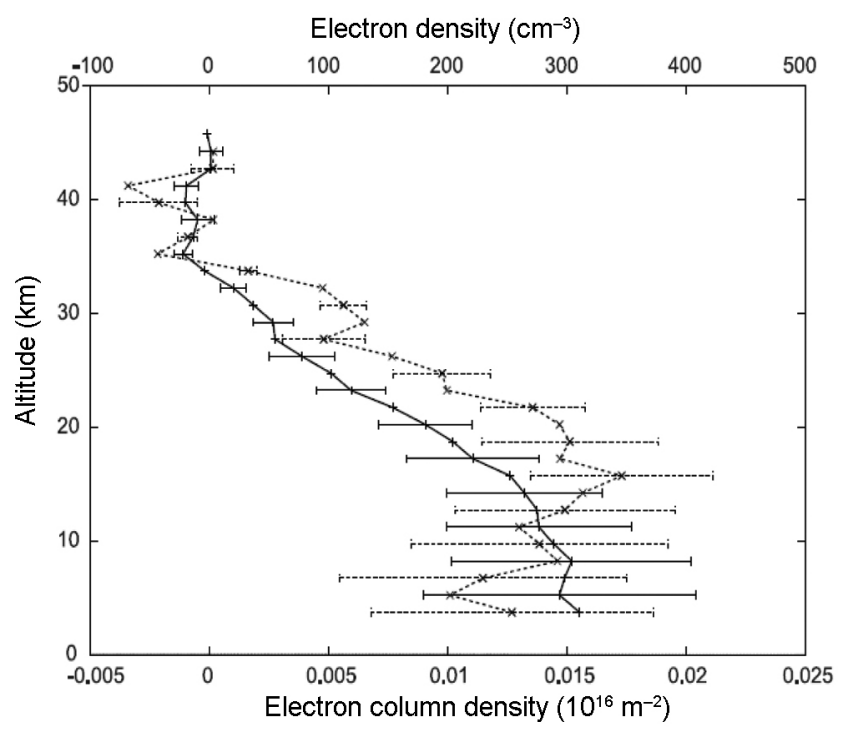

Figure 3. Altitude variation of electron density (dashed) and electron content (solid) of ionosphere ${ }^{14}$. 
extreme conditions, the dynamic range of $10 / \mathrm{cc}$ to $10^{4} / \mathrm{cc}$ was considered, while conceptualizing and developing the probe.

Depending on the solar elevation angle and activity conditions, the RAMBHA-LP probe can be inside or outside the photoelectron sheath. For higher electron densities the probe is expected to be outside the lunar plasma sheath and for lower electron density regime it is expected to be within the lunar plasma sheath. Any large change in the solar activity conditions during the mission duration will also result in the probe being inside or outside the sheath. Such a scenario would provide good understanding on the evolution of the near surface lunar plasma environment.

The electron temperature $\left(T_{\mathrm{e}}\right)$ is assumed to be $20,000 \mathrm{~K}$ respectively. The electronics is so designed that it caters to the wide dynamic range of $10^{6}$ obtained in the simulations. Based upon the simulations, it is inferred that the proposed LP for lunar ambience would work best for the specified operational parameters given in Table 1.

To cater to the full range of measurements in the lunar ionosphere, the probe voltage will sweep over a range of -12 to $12 \mathrm{~V}$ in steps of $0.1 \mathrm{~V}$ and back in the default mode. The duration of one sweep cycle will be $15 \mathrm{sec}$ in the default mode. The deduced characteristic curves will be used to estimate electron temperature and electron density near the lunar surface as explained in the previous section.

\section{Dynamic range}

The dynamic range of currents for which the probe is designed considering all regimes of operation and electron density from $10 / \mathrm{cc}$ to $10^{4} / \mathrm{cc}$ for $2.5 \mathrm{~cm}$ probe radius is given in Table 2 .

\section{Plasma ambience}

The Debye length $\left(\lambda_{\mathrm{D}}\right)$, the distance from the surface beyond which the sheath effects make no contribution, in any plasma is given by eq. (6)

Table 1. Operational parameters of probe in the default mode

\begin{tabular}{ll}
\hline Minimum sweep voltage (V) & $-12 \mathrm{~V}$ \\
Maximum sweep voltage (V) & $+12 \mathrm{~V}$ \\
Bias voltage steps (V) & $0.1 \mathrm{~V}$ \\
Step dwell time (ms) & $60 \mathrm{~ms}$ \\
Data sampling rate (ms) & $1 \mathrm{~ms}$ \\
Probe gain (feedback resistance) & $50 \mathrm{~K}, 1 \mathrm{M}$ and 20 M (rotation) \\
\hline
\end{tabular}

Table 2. Dynamic range of expected currents considering all regimes of operation and electron density from $10 / \mathrm{cc}$ to $10^{4} / \mathrm{cc}$ for $2.5 \mathrm{~cm}$ probe radius

\begin{tabular}{ll}
\hline Minimum current (A) & $25 \times 10^{-12}$ \\
Maximum current (A) & $25 \times 10^{-6}$
\end{tabular}

$$
\lambda_{\mathrm{D}}=k T_{\mathrm{e}} / n e^{2},
$$

where $k$ is the Boltzmann constant, $T_{\mathrm{e}}$ the electron temperature, $n$ the electron density and $e$ is the electronic charge.

Accordingly, the Debye length for plasma with $T_{\mathrm{e}}$ of $20,000 \mathrm{~K}$ and electron density in the range of $10 / \mathrm{cc}$ to $10^{4} / \mathrm{cc}$ is estimated to be in the range of $3 \mathrm{~m}$ to $\sim 10 \mathrm{~cm}$. As mentioned previously, the recent observations show that the lower limit of ionization at lower altitudes is $\sim 10^{2} / \mathrm{cc}$ The Debye length for electron density of $\sim 10^{2} / \mathrm{cc}$ is estimated as $\sim 0.98 \mathrm{~m}$. Hence a boom of $1 \mathrm{~m}$ length separating RAMBHA-LP probe from the Lander would cater to the normally expected electron density range.

\section{Payload subsystems}

The RAMBHA-LP payload consists of the mechanical system and the onboard electronics (Figure 4). The mechanical system consists of the spherical conducing $\mathrm{Ti}$ alloy probe attached to a non-conducing boom of $1 \mathrm{~m}$, a torsion spring based deployment mechanism, and a hold and release system. The probe will be in stowed position on the top deck of Chandrayaan-2 lander, and inside a dust protection system which protects the spherical probe from lunar dust, till it gets deployed in the lunar ambience. There is an onboard electronics system named as RAMBHA onboard controller (ROC), whose primary function is to control sensor voltage, data acquisition and spacecraft interface for telecommand (TC), telemetry (TM) and baseband data handling (BDH). ROC takes care of the data telecommand reception and execution, whenever a TC is sent from ground. It acquires the analog signals coming from the front end electronics (FEE) of the LP probe and converts it into digital domain using an ADC (24 bit). The necessary control signals to the probe are also provided by the onboard controller. The acquired data is packetized by the ROC system and then transferred to the spacecraft BDH system through LVDS interface in burst mode.

\section{RAMBHA-LP calibration methodology and results}

\section{Test methodology for probe calibration}

The calibration methodology is based on the premise that the LP can be characterized using the electrons emitted from the electron source in the vacuum environment. Nevertheless, a reference probe is needed. The RAMBHALP team, VSSC, has developed such a probe. Characterizing a probe in the controlled vacuum environment first, flying the same in sounding rockets/orbiters and calibrating the calculated electron density from the flight data using ground-based ionosonde measurements will provide 


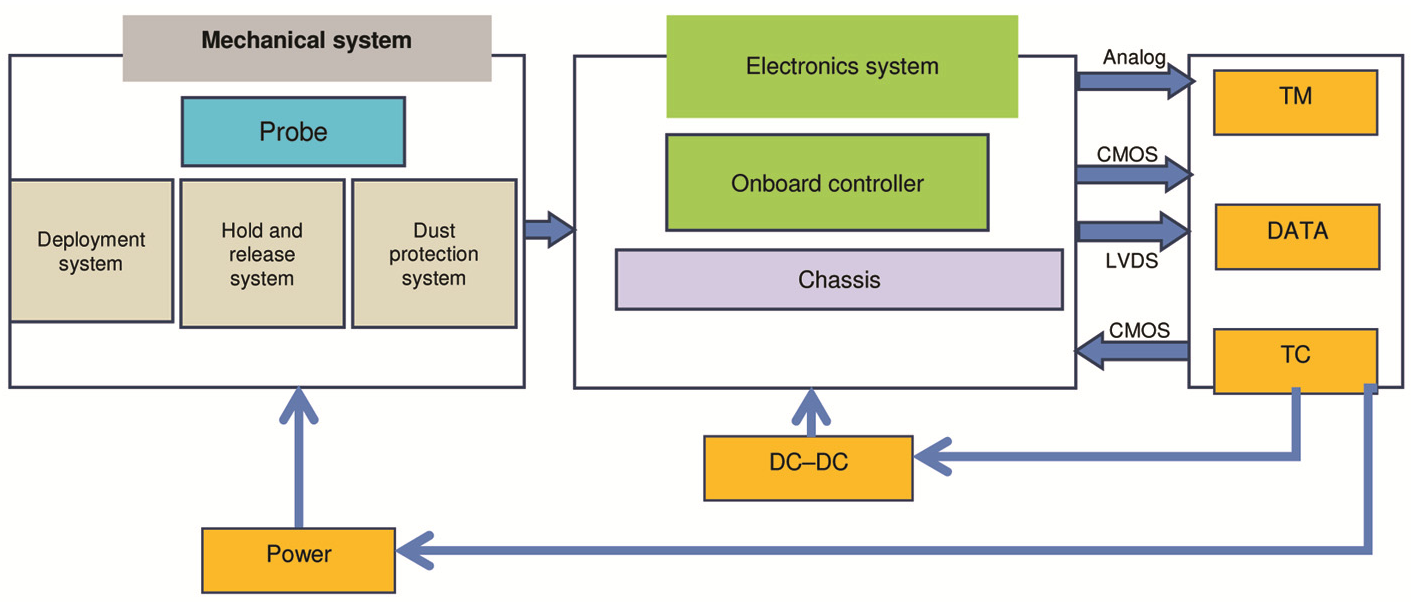

Figure 4. Block schematic of radio anatomy of Moon bound hypersensitive atmosphere and ionosphere-Langmuir probe.

a reference/standard for similar probes. The HVSSF, high vacuum space simulation facility, SPL, VSSC has a $1 \mathrm{~m}$ class vacuum chamber fitted with a calibrated high energy electron source. As per the methodology, another Langmuir probe which constituted one of the components of the ionization density and electric field analyser (IDEA) payload, is tested with electron source in the HVSSF. This spherical metallic Langmuir probe is of $2 \mathrm{~cm}$ diameter.

During the solar eclipse event of January 2010, the electron density and neutral wind_Langmuir probe (ENWi_LP) payload had been flown onboard ISRO's RH-300 rockets for measuring the eclipse induced changes in the electron densities and neutral winds ${ }^{24,25}$. In that campaign, the payloads profiled the ionosphere vertically over the Indian dip equatorial station, Trivandrum. The results from the ENWi component of the payload during the solar eclipse event are already reported ${ }^{24-26}$. On 23 June 2017, the same payload was operated in the electron density and electric field analyser mode as the IDEA payload and was positioned on the unique PS4 platform of the Indian Polar Satellite Launch Vehicle-C38 (PSLVC38) mission. As the PS4 platform took an altitudelatitude cut through the ionosphere, the payload sampled the bottom and topside ionospheres over a range of latitudes during the initial launch phase. The IDEA-LP was operated at a constant potential of $4 \mathrm{~V}$ and the current collected was measured using the payload electronics. The electron density $N_{\mathrm{e}}$, estimates were made from the measured currents. The measured $N_{\mathrm{e}}$ was calibrated using the electron density measured by a ground based ionosonde. The calibration factor $\left(k_{1}\right)$ so obtained is to be used for calibrating RAMBHA-LP. Prior to launch, the IDEA-LP payload had been subjected to characterization tests in the HVSSF at a pressure of $\sim 10^{-7}$ Torr. The test was done as described below:

A bias potential of $4 \mathrm{~V}$ was applied to the IDEA-LP probe and the current collected was measured using the proto electronics, for specific values of the electron beam energy of $4 \mathrm{eV}$ and source emission current of $1.2 \mu \mathrm{A}$. The offset of the proto electronics was determined a priori. The current collected by the probe was estimated from the measured output using the value of the feedback resistor according to Ohm's law.

If the current collected by the electronics after offset removal is $J_{\text {e-IDEA }}$

$$
J_{\text {e-IDEA }}=0.25 N_{\text {e-IDEA }} A_{1} e v_{\text {th }}(1+e V / k T),
$$

where $N_{\text {e-IDEA }}$ is the electron density sensed by IDEA-LP, $e$ is the electronic charge, $A_{1}$ is the probe surface area and $v_{\text {th }}$ is the mean thermal velocity of the impinging electrons. $V$ is the applied potential. Since $J_{\text {e-IDEA }}, e, A, v_{\text {th }}, k$ and $T$ are known, $N_{\mathrm{e}}$ can be estimated. This value of $N_{\text {e-IDEA }}$ is corrected using the estimated calibration factor of $k_{1}$ to obtain the corrected $N_{\mathrm{e}}\left(N_{\mathrm{e}-\text { IDEA-cor }}=k_{1} \cdot N_{\mathrm{e}-\text { IDEA }}\right)$.

\section{RAMBHA-LP probe calibration}

The test set-up used for the IDEA-LP and RAMBHA-LP probe calibration is shown in Figure 5. Here the probe alone is kept in the vacuum chamber and the electronics is kept outside. The proto board for RAMBHA-LP, enclosed in a chassis, has an I to $\mathrm{V}$ converter for the front end, followed by a differential amplifier to remove the bias.

The variable dc bias for the probe is provided from an external stable dc power source. The output from the proto electronics is taken through a screw terminal board, TBX, from which it can be read. A photograph of the RAMBHA-LP probe inside the high vacuum space simulation facility is shown in Figure 6.

The RAMBHA-LP is tested in two predefined conditions: (1) Fixed potential mode and (2) variable potential mode $( \pm 12 \mathrm{~V})$. In mode 1 a fixed potential of $4 \mathrm{~V}$ is applied to the probe and the current collected is measured 


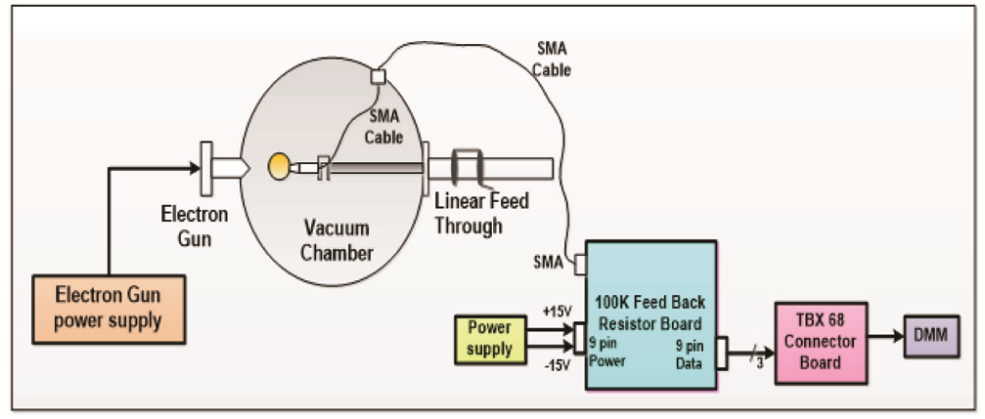

Figure 5. Test set-up for probe characterization.

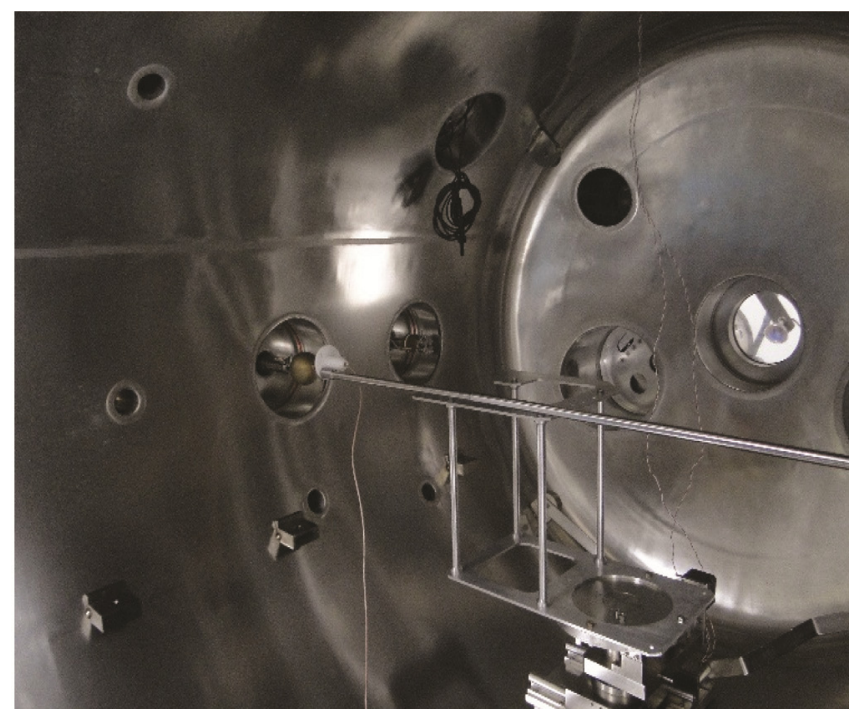

Figure 6. Test set-up for RAMBHA-LP inside the HVSSF chamber.

using the proto electronics for specific values of the electron beam energy of $4 \mathrm{eV}$ and source emission current of $1.2 \mu \mathrm{A}$. The electronics offset is measured a priori. The current collected by the probe is estimated from the measured output voltage as mentioned above using the value of the feedback resistor.

Here

$$
J_{\text {e-RAMBHA-LP }}=0.25 N_{\text {e-RAMBHA-LP }} A_{2} e v_{\text {th }}(1+e V / k T),
$$

where $J_{\text {e-RAMBHA-LP }}$ and $N_{\text {e-RAMBHA-LP }}$ are the current and electron density corresponding to the RAMBHA-LP payload, and $A_{2}$ corresponds to the RAMBHA-LP probe surface area. $V$ is the applied potential, $k$ the Boltzmann constant and $T$ is the temperature. The $N_{\text {e-RAMBHA-LP }}$ value can be estimated from the measured current using eq. (8).

As mentioned above, the IDEA-LP and RAMBHA-LP probes sense approximately the same background. Hence they should observe the same electron density once the calibration factor is accounted for. Hence RAMBHA-LP calibration factor $k_{2}$ is estimated as

$$
k_{2}=N_{\text {e-IDEA-cor }} / N_{\text {e-RAMBHA-LP. }}
$$

Keeping the electron beam energy and the emission current constant as above, the probe is given a variable bias potential from $-12 \mathrm{~V}$ to $+12 \mathrm{~V}$. The same electronics is used for current measurement. This helped to understand the response of the RAMBHA-LP probe to the varying bias voltage.

\section{Test equipment}

The various equipment used in the above configuration are listed: (i) Linear regulated dual output dc power supply, GST2505 with output voltage: $\pm 17 \mathrm{~V}, 100 \mathrm{~mA}$ with over voltage protection and fold back protection; (ii) DMM: 61/2 digits display; (iii) Electron source variable dc power source to provide the bias, with input current protection, and (iv) Data acquisition system and software in PC (optional).

\section{Test results}

\section{Test and calibration}

The RAMBHA-LP proto electronics is wired on the same PCB as that of IDEA-LP electronics, with two differences (i) The front end resistor is changed from $20 \mathrm{~K} \Omega$ to $100 \mathrm{~K} \Omega$, for better measurement accuracy in the vacuum environment and (ii) The probe bias is provided through an external dc power source instead of the constant bias derived internally.

\section{Test set-up for vacuum chamber test}

The test set-up as shown in Figure 5 is made for calibration of the probe. First, the continuity of the probe tip to the dc bias input is ensured before closing the chamber. The chamber is then closed and allowed to reach the desired vacuum level in the range of $10^{-7}$ Torr after which the electronics is powered from the power supply and the dc bias is kept at $-14 \mathrm{~V}$.

The electron source is switched on and after it stabilizes, the output voltage is noted. The dc bias for the probe is varied from $-12 \mathrm{~V}$ to $+12 \mathrm{~V}$ in pre-determined steps and the output voltage is noted in each case. The emission current 
of the source is varied from 1.2 to $1.4 \mu \mathrm{A}$ in steps and the above procedure is repeated for each configuration.

\section{RAMBHA-LP vacuum chamber test results}

As mentioned earlier, for given values of electron beam energy and the emission current, the probe is given a variable bias potential from $-12 \mathrm{~V}$ to $+12 \mathrm{~V}$. The output voltage is measured as seen by the proto electronics and the current collected is estimated using the value of the feedback resistor. Figure 7 shows the variation of the collected current as the bias is varied.

At relatively high negative potentials, a combination of ions (produced due to ionization of ambient neutrals with relatively lower ionization potentials) and electrons with higher thermal velocities is collected. As the negative repulsive potential is reduced, more electrons with lesser thermal velocities are able to reach the probe and this results in a steady increase in output voltage and therefore the current. When the applied potential turns from the negative to positive regime, the current collected increases with the increase in applied bias voltage. It is seen that, overall the probe behaves as expected for this configuration. The black arrow indicates the $4 \mathrm{~V}$ potential and the corresponding output voltage. The measurements made by the IDEA-LP and RAMBHA-LP at this bias voltage are used for cross calibration as described earlier.

The measured current varies in the range of $-2.3 \times 10^{-8}$ A to $9.5 \times 10^{-8} \mathrm{~A}$ for $1.2 \mu \mathrm{A}$ source emission current while the same varies in the range of $-2.6 \times 10^{-8} \mathrm{~A}$ to $1.1 \times 10^{-7} \mathrm{~A}$ for $1.4 \mu \mathrm{A}$ source emission current (Figure 7). The arrow indicates the $4 \mathrm{~V}$ potential and the corresponding output current. The measurements made by the IDEA-LP and RAMBHA-LP at this bias voltage are used for cross calibration.

\section{Estimation of plasma temperature and density from vacuum chamber test}

The current voltage characteristics depicted in Figure 7 are used to estimate the plasma parameters. The characteristic curve shows zero current collection around

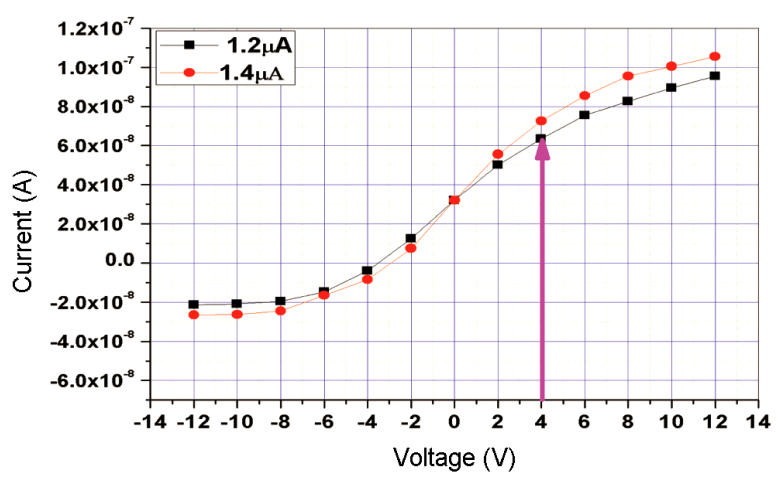

Figure 7. Variation of current collected with bias voltage during vacuum chamber test.
$-2.8 \mathrm{~V}$ bias potential. This will be the floating potential of the probe. The slope of the voltage versus natural logarithm of current curve is taken for the retarding potential region between the floating potential and the point in the electron saturation region prior to the one where the slope of the curve changes. This slope is related inversely to the electron temperature in $\mathrm{eV}$. Hence electron temperature is determined from the two curves in Figure 7 for the two values of emission current. The estimated temperature in each case is used to get the thermal velocity using the expression $v_{\mathrm{th}}=\left(8 \mathrm{kT} / \pi m_{\mathrm{e}},\right)^{0.5}$ where $k$ is the Boltzmann constant, $T$ the electron temperature and $m_{\mathrm{e}}$ is the electronic mass. The electron density $N_{\mathrm{e}-\mathrm{RAMBHA}-\mathrm{LP}}$ is then calculated from the eq. (8).

As mentioned previously, $k_{2}$ the calibration factor of RAMBHA-LP is estimated from eq. (9) using the estimated $N_{\text {e-IDEA_cor }}$ and $N_{\text {e-RAMBHA-LP. }}$ The estimated calibration parameters for each of the emission current values for RAMBHA-LP are given in Table 3.

\section{RAMBHA onboard controller performance during current source tests}

The RAMBHA-LP onboard controller (ROC) is fed current directly from a current source through a double shielded cable. The bias voltage sweep (from -12 to $-12 \mathrm{~V}$ is applied in variable steps) from the DAC of ROC is also applied through the same cable. In this configuration which simulates the actual experimental condition the output of the ROC ADC is to be monitored to examine: (i) the functioning of ROC and (ii) the effect of the applied bias on the sensed current.

First, the power supply is switched on and the offset, if any is noted. The bias power source is switched on with $0 \mathrm{~V}$ output. The current source is switched on and varied in steps from $50 \mathrm{nA}$ to $20 \mu \mathrm{A}$, since both ion and electron currents are expected in the lunar ambience. The output current at each value of applied bias voltage is acquired as the voltage sweep is given (from $-12 \mathrm{~V}$ to $12 \mathrm{~V}$ ) for each value of input current. This exercise is carried out for all the three gain resistors $(50 \mathrm{~K}, 1 \mathrm{M}$ and $20 \mathrm{M})$. It is observed that there exists a unique response for each resistor to a given input current as the bias voltage sweep is applied.

\section{ROC characterization using current source based test results}

Figure 8 depicts the ROC output voltages for given electron and ion current inputs for the three resistors,

Table 3. Calibration parameters for different emission currents

\begin{tabular}{lc}
\hline Emission current $(\mu \mathrm{A})$ & $\begin{array}{c}\text { RAMBHA-LP calibration } \\
\text { parameter (electrons/cc/pA) }\end{array}$ \\
\hline 1.2 & 1.3 \\
1.4 & 1.1 \\
\hline
\end{tabular}



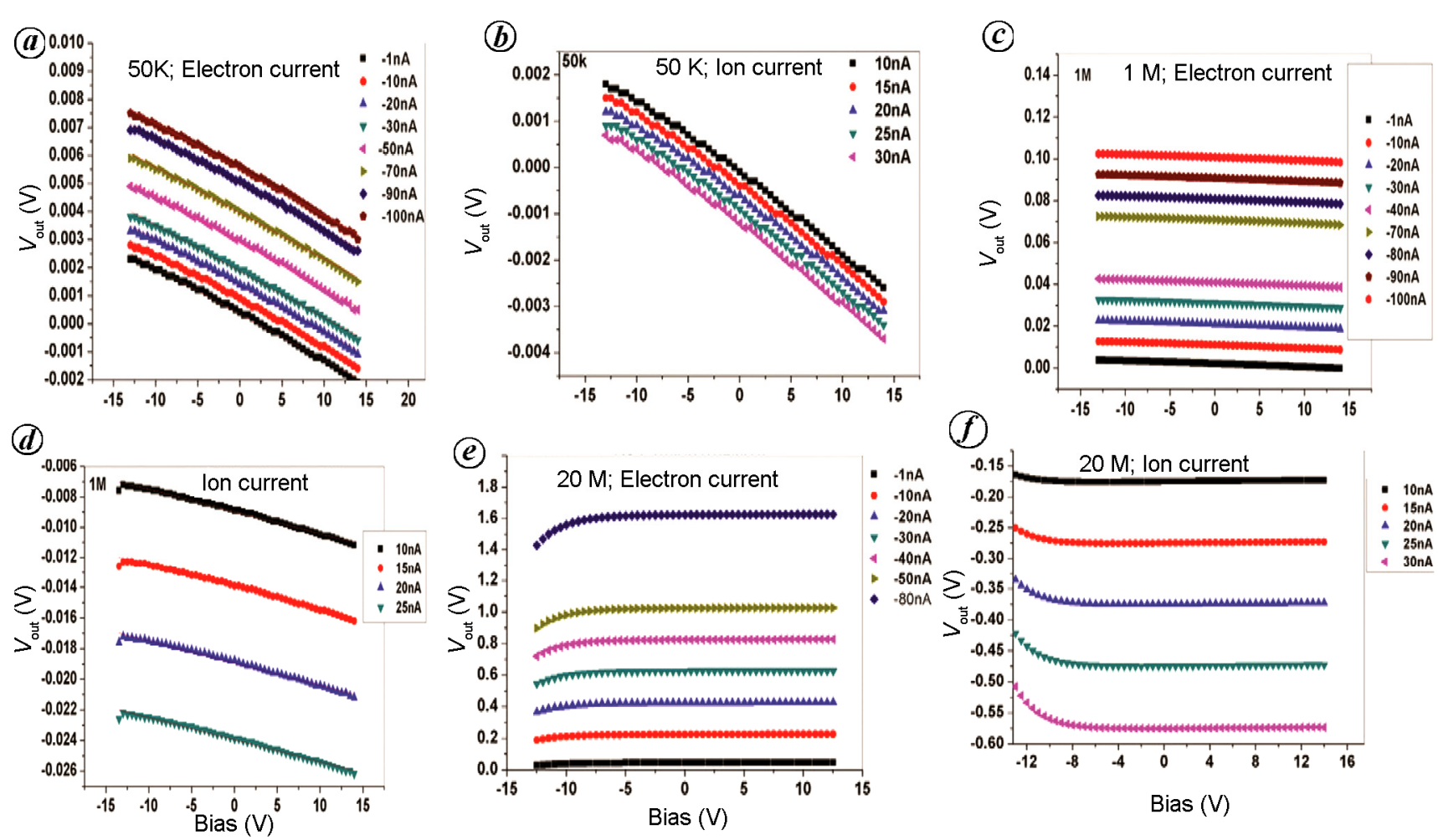

Figure 8. RAMBHA onboard controller output voltages for given electron and ion current inputs for the three resistors, $50 \mathrm{~K}, 1 \mathrm{M}$ and $20 \mathrm{M}$.
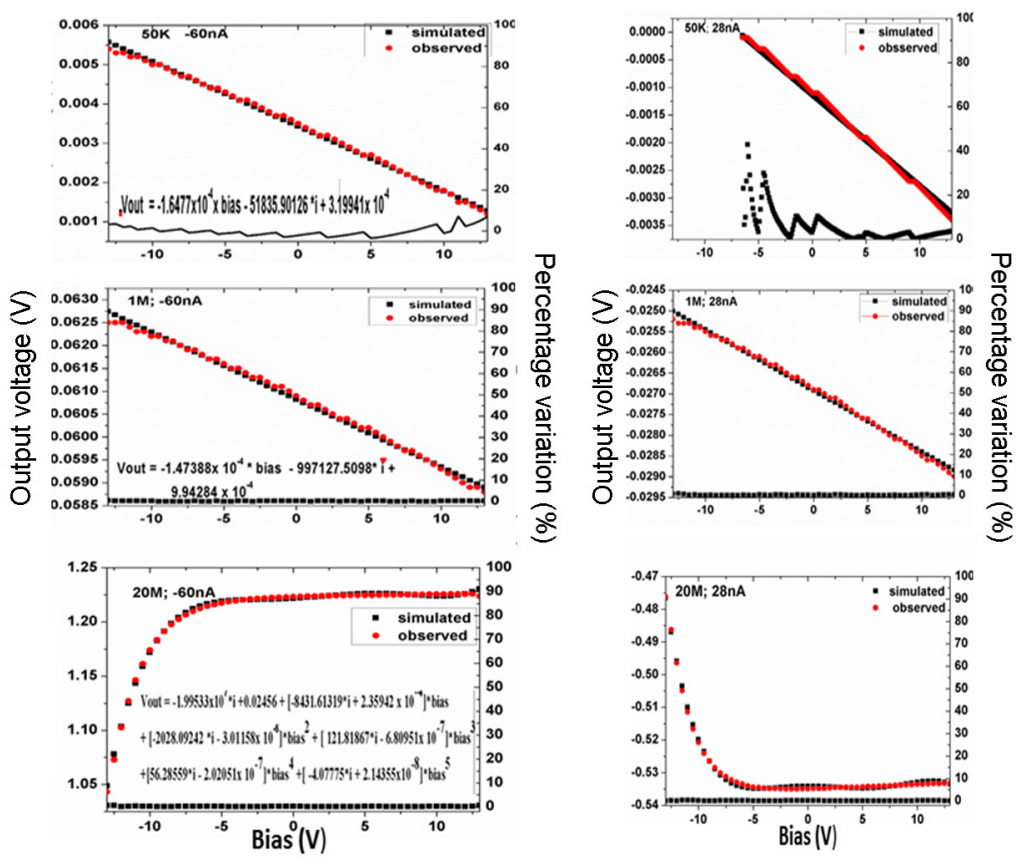

Figure 9. Variation of simulated and observed output voltages as a function of bias voltage for $60 \mathrm{nA}$ electron current and $28 \mathrm{nA}$ ion current.

$50 \mathrm{~K}, 1 \mathrm{M}$ and $20 \mathrm{M}$. Panels $(a),(c)$ and (e) show the ouptput voltages for given input electron currents corresponding to the $50 \mathrm{~K}, 1 \mathrm{M}$ and $20 \mathrm{M}$ resistors respectively. The variations are linear for $50 \mathrm{~K}$ and $1 \mathrm{M}$ resistors while they are non-linear for the $20 \mathrm{M}$ resistor. Panels $(b),(d)$ and $(f)$ show the the ouptput voltages for given input ion currents corresponding to the $50 \mathrm{~K}, 1 \mathrm{M}$ and $20 \mathrm{M}$ resistors respectively. Here also, the variations are linear for $50 \mathrm{~K}$ and $1 \mathrm{M}$ resistors while they are nonlinear for the $20 \mathrm{M}$ resistor. A systematic change in the output voltages is observed in all panels as the input current changes. Linear fits are given to the linear variation of output voltage given in panels $(a)$ and $(b)$ for the different input current values. It is found that the intercept for the linear fit expressions have direct dependence on current and this dependence is deciphered 
and substituted in the original linear fit to obtain the generalized expression for the output voltage for any given input voltage. The same approach is adopted for $1 \mathrm{M}$ resistor also. In the case of $20 \mathrm{M}$ resistor, a poynomial of the form $y=a+b x+c x^{2}+d x^{3}+e x^{4}+f x^{5}$ is found to be the best fit expression to the variations of output voltage. The dependence of the coefficients of the expression on the input current is deciphered and substituted in the generalized expression.

Figure 9 shows the variation of simulated and observed output voltages as a function of bias voltage for $60 \mathrm{nA}$ electron current and $28 \mathrm{nA}$ ion current, for the $50 \mathrm{~K}$ (top 2 panels), $1 \mathrm{M}$ (middle 2 panels) and $20 \mathrm{M}$ resistors (bottom 2 panels). It is readily seen that the model is able to readily give the output voltages for given input currents. The percentage variation of the simulated currents from the observed currents is less than $10 \%$ for all cases except for the negative bias voltages below $-3 \mathrm{~V}$ for $28 \mathrm{nA}$ ion current where the percentage variation goes to as high as $40 \%$. Thus overall the input current can be modelled using the derived expressions.

\section{Conclusion}

The RAMBHA-LP probe has been designed and developed in-house. It will be flown onboard CH-2 Lander to the Moon. The probe has been calibrated and deemed fit for measuring the plasma density of the tenuous lunar ionized environment near the surface. The RAMBHA-LP payload will be making lunar near-surface plasma measurements for the first time in situ at the near polar regions of the Moon. The payload measurements in this unexplored region of the Moon will certainly give new insights into the heterogeneity of the lunar near-surface plasma environment vis-á-vis the equatorial measurements made during previous lunar missions.

1. Freeman Jr, J. W. and Hills, H. U., Positive ions at the Apollo 12 ALSEP site resulting from the Apollo 13 S-IV B Impact. EOS, 1970, 51, 821

2. Freeman Jr, J. W., Energetic ion bursts on the nightside of the Moon. J. Geophys. Res., 1972, 77(1), 239-243.

3. O'Brien, B. J. and Reasoner, D. L., Charged particle lunar environment experiment, Apollo 14 Preliminary Science Report, NASA Spec. Publ., 1971, 272, 193.

4. Neugebauer, M., Snyder, C. W., Clay, D. R. and Goldstein, B. E., Solar wind observations on the lunar surface with the Apollo-12 Alsep. Planet. Space Sci., 1972, 20, 1577.

5. Berg, O. E., Richardson, F. F. and Burton, H., Lunar ejecta an meteorites experiment. In APOLLO 17 Preliminary Science Report, NASA SP-330, 1973, p. 16.

6. Berg, O. E., Richardson, F. F., Ree, J. W. and Auer, S., Preliminary results of a cosmic dust experiment on the Moon. Geophys. Res. Lett., 1974, 1(7), 289-290; doi:10.1029/GL001i007p00289.

7. Reasoner, D. L. and Burke, W. J., Characteristics of the lunar photoelectron layer in the geomagnetic tail. J. Geophys. Res., $1972,77,6671$.

8. Reasoner, D. L. and Burke, W. J., Direct observations of the lunar photoelectron layer. Proceedings of the Third Lunar Science Conference, Supplement 3, Geochim. Cosmochim. Acta, 1972, 3, 2639.
9. Rich, F. J., David, L, Reasoner and William, J. B., Plasma sheet at lunar distance: characteristics and interactions with lunar surface. J. Geophys. Res., 1973, 78, 8097.

10. Savich, N. A., Lunar plasma model. Space Res., 1976, 16, 941-943.

11. Feuerbacher, B., Anderegg, M., Fitton, B., Laude, L. D. and Willis, R. F., Photoemission from lunar surface fines and the lunar photo electron sheath. Proceedings of the third lunar science conference (Supplement 3, Geochim. Cosmochim. Acta), The MIT Press, 1972, 3, 2655-2663.

12. Willis, R. F., Anderegg, M., Feuerbacher, B. and Fitton, B., Photoemission and secondary electron emission from lunar surface material. In Photon and Particle Interactions With Surfaces in Space (eds Grard, R. J. L. and Reidel, D.), Dordrecht, The Netherlands, pp. 389-401.

13. Sternovsky, Z., Chamberlin, P., Horanyi, M., Robertson, S. and Wang, X., Variability of the lunar photoelectron sheath and dust mobility due to solar activity. J. Geophys. Res., 2008, 113, A10104; doi:10.1029/2008JA013487.

14. Imamura, T. et al., Radio occultation measurement of the electron density near the lunar surface using a subsatellite on the SELENE mission. J. Geophys. Res., 2012, 117, A06303; doi:10.1029/ 2011JA017293.

15. Vyshlov, A. S., Preliminary results of circumlunar plasma research by the Luna 22 spacecraft. Space Res., 1976, 16, 945-949.

16. Vyshlov, A. S., Savich, N. A., Vasilyev, M. B., Samoznaev, L. N., Sidorenko, A. I. and Shtern, D. Y., Some results of cislunar plasma research. NASA Tech. Rep., 1976, 397, 81-85.

17. Stern, S. A., The lunar atmosphere: history, status, current, problems, and context. Rev. Geophys., 1999, 16, 941-943.

18. Choudhary, R. K., Ambili, K. M., Choudhury, S., Dhanya, M. B. and Bhardwaj, A., On the origin of the ionosphere at the Moon using results from Chandrayaan-1 $\mathrm{S}$ band radio occultation experiment and a photochemical model. Geophys. Res. Lett., 2016, 43, 10025-10033; doi:10.1002/2016GL07061.

19. Langmuir, I. and Mott-Smith, H., The theory of collectors in gaseous discharges. Phys. Rev., 1926, 28, 727-763.

20. Spencer, N. W., Brace, L. H., Carignan, G. R., Taeusch, D. R. and Niemann, H., Electron and molecular nitrogen temperature and density in the thermosphere. J. Geophys. Res., 1965, 70(11), 2665-2698.

21. Prakash and Subbaraya, Langmuir probe for the measurement of electron density and electron temperature in the ionosphere, Rev. Sci. Instrum., 1967, 38(5), 1132-1136.

22. Smith, L. G., Small rocket instrumentation techniques (ed. Maeda, K. I.), North Holland Publishing, Amsterdam, 1969, p. 1.

23. Stubbs, T. J., Glenar, D. A., Farrell, W. M., Vondrak, R. R., Collier, M. R., Halekas, J. S. and Delory, G. T., On the role of dust in the lunar ionosphere. Planet. Space Sci., 2011, 59, 1659-1664.

24. Manju, G. et al., A Novel probe for in situ electron density and neutral wind (ENWi) measurements in the near Earth space. J. Atmos. Sol. Terr. Phys., 2012, 74, 81-86.

25. Manju, G., Sridharan, R., Sudha Ravindran, Madhav Haridas, M. K., Pant, T. K., Sreelatha, P. and Mohan Kumar, S. V., Rocket borne in situ electron density and neutral wind measurements in the equatorial ionosphere results from the January 2010 annular solar eclipse campaign from India. J. Atmos. Sol.-Terr. Phys., 2012, 86, 56-64.

26. Manju, G., Madhav Haridas, M. K., Ramkumar, G., Pant, T. K., Sridharan, R. and Sreelatha, P., Gravity wave signatures in the dip equatoria ionosphere-thermosphere system during the annular solar eclipse of 15 January 2010. J. Geophys. Res.: Space Phys., 2014, 19, 4929-4937; http://dx.doi.org/10.1002/2014JA019865.

Received and accepted 27 August 2019

doi: $10.18520 /$ cs/v118/i3/383-391 